\title{
LA HISTORIA ECONOMICA DE LA EMPRESA COMO DISCIPLINA INDEPENDIENTE: UNA PERSPECTIVA HISTORICA
}

PEDRO FRAILE

Universidad Carlos III de Madrid

La naturaleza metodológica de la Historia Económica como economía aplicada raramente se manifiesta tan evidente como en el campo de la Historia Económica de la empresa. Lo que para la Historia Económica general es un signo de identidad adquirido a lo largo de muchos años, para la Historia Económica de la empresa fue la razón misma de su aparición como disciplina independiente. Hace ya 65 años, la necesidad de ofrecer un campo de «experimentación» para las técnicas de dirección empresarial llevó a Wallace $B$. Donham, decano de la Escuela Graduada de Comercio de la Universidad de Harvard a establecer en 1927 la cátedra Straus de Historia Económica de la empresa con la idea de que, en sus propias palabras, una figura acádemica distinguida

se uniese al claustro de Harvard para ver si en su Escuela Graduada de Comercio fuese posible poner en marcha un programa de investigación y enseñanza en el campo de la Historia Económica general y de la empresa que estuviese tan intimamente relacionado con los problemas reales del hombre de empresa que llegase a ser una auténtica contribución social ${ }^{1}$.

Donham habia fundado en 1925 la Sociedad Histórica de Empresas (Business Historical Society) y estaba convencido de que el estudio de situaciones empresariales del pasado podria ser un complemento útil para el «método de casos» en la enseñanza de las técnicas empresariales. Era obvio que la Historia Económica general no podia cumplir esta función para los alumnos de la Escuela Graduada de Comercio de la Universidad de Harvard, en la cual se ha-

1 Citado por Fritz Fedlich (1962), p. 62. 
bia seguido siempre una aproximación empirica desde su fundación en 1908. La misión de articular una nueva disciplina que mostrase en perspectiva histórica los problemas de dirección empresarial recayó en Norman S. B. Gras, pionero de la «business history» y profesor en la Universidad de Minnesota desde 1918. Gras habia estudiado el papel de las empresas en el desarrollo regional del norte de los Estados Unidos, y había creado un grupo de trabajo con sus alumnos dedicado al análisis de casos específicos de empresas regionales. Gras permaneció en Harvard hasta su jubilación en 1950 y, junto con Edwin F. Gay, fue corresponsable del establecimiento de Harvard como plataforma inicial de la Historia Económica de la empresa. Edwin F. Gay fue durante un tiempo decano de la Escuela y colaboró como director desde 1928 hasta 1932 en la primera revista profesional con el nombre de la nueva disciplina: The Joumal of Economic and Business History ${ }^{2}$.

A pesar de que la trayectoria académica de la Historia Económica de la empresa como disciplina independiente empezó en la década de 1920, sus raices $y$ antecedentes se remontan al siglo xix. La primera monografía histórica de una empresa fue publicada en 1825 para conmemorar el centenario de la Siderurgia Lauchhammer en Sajonia ${ }^{3}$. Sin embargo, el interés por las unidades de producción como protagonistas históricos está asociado a dos escuelas de finales del siglo XIX y principios del XX. Una es la tradición «anti-empresa» de los historiadores económicos de Inglaterra, como Toynbee, Hobson o el matrimonio Webb, y de los Estados Unidos, como Thorstein Veblen, cuyo Theory of Business Enterprise (1904) identifica la imagen del empresario con la de un «malvado avaro dispuesto a explotar todos los intersticios de la tecnologia industrial para sus propios y egoístas fines» 4.

El otro gran antecendente de la Historia Económica de la empresa fue la escuela histórica alemana. Su método inductivo desafiaba las generalizaciones de los clásicos y se dirigía al estudio de todos los segmentos de la sociedad y su historia. Esta búsqueda llevó a Gustav Schmoller al estudio de la empresa. Su «Die geschichtliche Entwicklung der Unternehmung» (1890-93) es una serie de articulos sobre el desarrollo histórico de empresas locales, y en Grundiss der allgemeinen Volkswirtschaftslebre (1901) trata en profundidad el proceso histórico del cambio en la organización empresarial. El interés de Karl Bücher por las etapas del crecimiento estaba basado en la importancia que concedia al cambio regional y sectorial y dio lugar a numerosos estudios de sectores industriales y empresas concretas. Aunque no pertenecia a la escuela histórica, Wer-

2 Ibid, p. 63.

3 Ibid., p. 61.

4 Clarence C. Walton (1962), p. 28. 
ner Sombart contribuyó al establecimiento de Alemania como el centro original del estudio histórico de la empresa y el empresario. Su Der Moderme Kapitalismus (1902) y Der Bourgeois (1913) hacen hincapié en el desarrollo histórico del «espiritu de empresas como factor psicológico fundamental de la evolución del capitalismo.

La influencia alemana fue especialmente importante en Inglaterra y en los Estados Unidos. George Unwin tenía experiencia directa de la industria británica y su organización. Su Industrial Organization in the Sixteenth and Seventeentb Centuries (1904) analiza la evolución de la estructura de mercados desde la ruptura de los gremios medievales ingleses hasta la aparición del capitalismo comercial e industrial moderno, y en Samuel Oldknow and the Arkwrights. The Industrial Revolution at Stockport and Marple (1924) estudia el sector textil en la Revolución Industrial a partir de archivos empresariales. Unwin estudió en Cardiff y en Oxford, y en 1898 consiguió una beca para ampliar estudios en Berlín. Su contacto con Schmoller le llevó a la idea de fundar una escuela de Historia Económica en Gran Bretaña que emulase las enseñanzas de los alemanes'. Tras volver a su pais, comenzó una larga carrera docente que le llevó a la London School of Economics, la Universidad de Edimburgo y la Universidad de Manchester. En esta última pasó catorce años, durante los cuales su investigación y docencia tuvo una marcada influencia sobre alumnos tan destacados como G. W. Daniels, T. S. Ashton y C. Gill, que formaron el núcleo de la siguiente promoción en la nueva disciplina ${ }^{6}$.

$\mathrm{El}$ contacto de muchos jóvenes economistas norteamericanos con universidades y profesores de Alemania canalizó la influencia de la escuela histórica hacia los claustros de los Estados Unidos a partir de los años 1880. Los «Socialdemócratas Americanosw, como los denominó Henrietta Larson 7, fueron muy influyentes en la fundación en $\mathbf{1 8 8 5}$ de la American Economic Association, entre cuyos objetivos estaba promocionar la regulación estatal de las empresas americanas. El interés por la intervención reguladora llevó a economistas como John $\mathrm{R}$. Commons al estudio de estructuras concretas de mercado con el fin de denunciar situaciones monopolísticas que precisaban, en su opinión, de la intervención correctora del Estado. Sin embargo, y a diferencia de sus predecesores alemanes, los institucionalistas norteamericanos se interesaban más por el impacto social de los monopolios que por los aspectos internos de las empresas monopolisticas ${ }^{8}$.

5 R. H. Tawney (1966), p. xxii.

Ibid, pp. xxvi-lxi.

7 Henrietta M. Larson (1939), p. 15.

- Ibid, pp. 15-16. 
La conexión más fructifera desde el punto de vista de la Historia Económica de la empresa fue la que se estableció entre el norteamericano Edwin Gay y su maestro alemán Schmoller, de la Universidad de Berlín. Como en el caso del británico George Unwin, el americano Edwin Gay también se oponía a la tendencia intervencionista alemana, pero compartía el método inductivo de Schmoller y su énfasis en la recogida y observación de los datos como etapa inicial de la investigación. Como profesor de Historia Económica, Gay tuvo una influencia decisiva sobre sus alumnos. La tendencia de Gay como historiador económico cuantitativo se hizo sentir claramente sobre Wesley C. Mitchell, y su inclinación por la Historia Económica aplicada influyó sobre Norman Gras. Este último había estudiado a Karl Bücher, Werner Sombart y George Unwin, y bajo la dirección de Gay aprendió la importancia estratégica de las empresas en el cambio económico?

La adscripción de Gay y Gras a la Escuela Graduada de Comercio y su colaboración en el Joumal of Economics and Business History convirtió a Harvard en el primer centro académico donde los historiadores económicos de la empresa se configuraron como un grupo independiente con personalidad propia. Nombres como Ralph M. Hower, Edward Edelman, Kenneth W. Porter, Charles W. Moore, J. Owen Stalson y Charles S. Popple contribuyeron al desarrollo del proyecto inicado por Gras, y establecieron el método de «estudio de casos» como el signo distintivo de la Historia de la empresa en Harvard. Sin embargo, ninguno de ellos destacó tanto como Henrietta Larson. Alumna de Gras en sus cursos de Historia Económica en Minnesota, Larson llegó a Harvard casi al mismo tiempo que Gras y fue su colaboradora en los proyectos más importantes, especialmente en la preparación de casos de historias empresariales. Además de sus estudios sobre la Standard Oil Company publicados durante los años 1950 y 1960, Larson fue coautora, con Gras, de Casebook in American Business History (1939), que junto con la obra más conocida de Gras, Busines and Capitalism, publicado el mismo año, se convirtieron en los textos básicos del momento para la nueva disciplina ${ }^{10}$.

La posición de Harvard se vio reforzada por la presencia de Joseph A. Schumpeter y Arthur H. Cole. Aunque su interés se centraba en la actividad empresarial más que en las empresas mismas, su influencia en el desarrollo del nuevo campo fue decisiva ". Con la ayuda financiera de la Fundación Rockefeller, ambos pusieron en marcha en 1948 el Centro de Investigaciones de Historia Empresarial, que dirigiria Cole. Hasta su clausura, diez años más tarde, el

- Ibid, pp. 16-17.

10 Ralph W. Hidy (1962), p. 7.

"Rafael Castejón (1983), pp. 162-163. 
Centro se convirtió en punto de encuentro y foro de autores como Thomas C. Cochran, J. W. Flin, A. Gerschenkron, B. F. Hoselitz, D. S. Landes, P. Mathias, D. C. North, F. Redlich, A. Sapori, H. J. Habakkuk y R. E. Cameron, que a través de la publicación del Centro, Explorations in Entrepreneurial History, contribuyeron a destacar el papel de los empresarios y las empresas en la Historia Económica general. De hecho, las investigaciones del Centro tendieron a fundirse, durante los años cincuenta, con las del grupo de Gras y a incorporar métodos de otras disciplinas, como el crecimiento económico o la sociología, para dar nuevos resultados como, por ejemplo, el trabajo de Everett E. Hagen, On the Theory of Social Change: How Economic Growth Begins (1962) ${ }^{12}$.

La Historia Económica de la empresa empezó a desarrollarse casi simultáneamente en Gran Bretaña, pero, como se ha indicado antes, la tradición británica en la nueva disciplina venía, por supuesto, de muy lejos. En 1951 el Business Archives Council de Gran Bretaña poseía una colección de más de quinientas historias de empresas ${ }^{13}$. Como se ha mencionado antes, la trayectoria de George Unwin, continuó durante la entreguerra. Los trabajos de G. W. Daniels, The Early English Cotton Industry (1920); H. Hamilton, The English Copper and Brass Industries to 1800 (1926); J. U. Nef, The Rise of the British Coal Industry (1932); T. S. Ashton, An Eighteenth Century Industrialist: Peter Stubs of Wamington 1756-1806 (1939); sir John Clapham, The Bank of England (1944); D. L. Burn, The Economic History of Steel-Making 1867-1929 (1940); G. C. Allen, British Industries and their Organization (1933); G. Gill, The Rise of the Irish Linen Industry (1925), y muchos otros más habían explorado algunos sectores industriales y comerciales antes de 1950 . En estas circunstancias, la obra de Gras y Cole encontró rápidamente una continuación en Gran Bretaña durante los años 1950 y 1960. La obra de Charles Wilson History of Unilever se publicó con un enorme éxito en 1954 y constituyó el principio de

una permanente preocupación por la empresa, no simplemente en su acepción común de aempresa privadaw, sino como proyecto humano con el logro individual como elemento vital de motivación en la historia ${ }^{14}$.

En 1954 empezó a publicarse en Harvard la Business History Review ${ }^{15}$. Tan

12 Ralph W. Hidy (1968), p. 477.

13 T. C. Barker, R. H. Campbell y P. Mathias (1960), pp. 24-25.

I4 D. C. Coleman y Peter Mathias (1984), p. viii.

15 La Business History Review aparecia como continuación del Bulletin of the Business Historical Society que se publicaba en Harvard desde 1926. A este respecto, Barry Supple señala que los historiadores económicos de la empresa dispusieron en los Estados Unidos de un órgano propio mucho antes que los historiadores económicos generales. El Jourmal of Economic History empezó su publicación en 1941. Ver B. E. Supple (1958), p. 66. 
sólo unos años después, en 1958, la Universidad de Liverpool empezó a publicar Business History bajo la dirección de Francis Hyde, y con la ayuda de historiadores de la empresa de la talla de S. B. Saul, Sheila Marrimer o Charles Wilson, entre otros. Uno de los alumnos de Wilson, William Reader, publicó historias empresariales de Imperial Chemical Industries, Metal Box, Bowater y el Grupo Weir. Otro, Ronald Ferrier, estudió la historia de British Petroleum; otros muchos se ocuparon más tarde de otras muchas empresas y sectores británicos, y más recientemente, Leslie Hanna empezó a aplicar el modelo chandleriano al caso británico ${ }^{16}$.

La enseñanza e investigación de la disciplina en Gran Bretaña se generalizó casi tanto como en los Estados Unidos durante las décadas de 1970 y 1980. La Business History Unit (BHU) se fundó en la London School of Economics en 1978. Otros centros aparecieron a continuación: el Center for Business History se fundó en el Departamento de Ciencias Políticas e Historia de Coventry Polytechnic en 1984 y la Escuela de Comercio de Cardiff estableció en 1988 la Business History Research Unit. No obstante, el avance más importante fue la fundación en 1987 del Center for Business History Research de la Universidad de Glasgow, que comparte ahora con la LSE el liderazgo británico en este campo ${ }^{17}$. Otras universidades como Manchester, Reading, Bristol Polytechnic, Lancaster, East Anglia, Edimburgo y Brunel han puesto también en marcha centros para el estudios de la Historia Economica de la empresa donde se vienen desarrollando la investigación y la docencia de la disciplina como parte integral de sus programas desde hace ya bastantes años ${ }^{18}$.

La actividad investigadora se extendió a toda Europa y Japón durante los años 1960 y 1970. Trabajos tales como J. P. McKay, Pioneers for Profit: Foreign Entrepreneurship and Russian Industrialization (1970), o Peter Hertner, Il capitale tedesco in Italia dall Unita alla prima guerra mondiale (1984), contribuyeron a que la disciplina se fuera extendiendo por todos los paises europeos continentales, y a que grandes empresas como Siemens, Renault, los Chemin de Fer du Nord, Swedish Match, Mitsui y Mitsubishi fuesen investigadas y sus respectivas historias escritas y publicadas ${ }^{19}$. Al mismo tiempo, se establecieron sociedades de Historia Económica de la empresa en Japón y Alemania y, al lado de las prestigiosas Business History y Business History Review, aparecieron otras publicaciones profesionales como, por ejemplo, Histoire des Enterprises en Francia, y Tradition, Zeitschrift für Firmengeschichte und Untermebmerbiographie en Alemania.

\footnotetext{
16 Leslie Hanna (1976).

17 Charles Harvey y Geoffrey Jones (1990), pp. 8-10.

18 Ibid, p. 11.

19 Alfred D. Chandler (1984), pp. 6-7.
} 
Bajo la dirección de Gras, Gay, Larson y Wilson como autores más influyentes, la Historia Económica de la empresa siguió fundamentalmente el método del estudio de casos concretos de empresas. Pero en realidad la aproximación simplemente descriptiva de la disciplina empezó desde muy pronto a ser insuficiente ${ }^{20}$. En Business and Capitalism (1939) el mismo Gras hizo ya un esfuerzo por relacionar la evolución y las etapas del capitalismo -pequeño capitalismo, capitalismo mercantil, capitalismo industrial, capitalismo financiero y capitalismo nacional - con los sistemas empresariales y tipos de empresas predominantes en cada una de ellas. No obstante, el estudio pormenorizado de casos concretos, la historia «de empresas» (company bistory), siguió siendo el método habitual hasta los años 1970 en la Historia Económica de la empresa. Los debates se centraban casi siempre en aspectos algunas veces de tipo ético - los empresarios como «robber barons»- y otras veces de tipo ideológico, tales como el papel de los empresarios en su relación con la sociedad.

La oposición al uso explícito de teoría económica encontró los mismos obstáculos que en la Historia Económica general. En «Economic Theory and Business History", Francis Hyde argumentaba que la «Teoria económica no es un patrón universal de medida que pueda ser usado indiscriminadamente por el historiador" ${ }^{21}$. Sin embargo, durante la posguerra la historia de empresas habia entrado ya en una etapa de rendimientos decrecientes. En 1960 Arthur M. Johnson se refería a la metodología de la Historia Económica de la empresa:

La historia de cualquier tipo, para ser historia, tiene que tener un gran contenido empirico, y la Historia Económica de la empresa no es excepción. De hecho aún se necesitan datos empiricos básicos en muchas partes de nuestra disciplina. Sin embargo, existe una necesidad aún más urgente: recapacitar, sintetizar y conceptualizar sobre la base del trabajo pionero que ya se ha realizado. Mientras seguimos aumentando la masa de datos debemos también encontrar la manera de abrirnos paso a través de ella 22 .

A partir de los años 1960 la disciplina cambió su énfasis y enfoque desde una perspectiva de la «historia de empresas» a otra de «las empresas en la historia» ${ }^{23}$. En palabras de Chandler, la Historia Económica de la empresa «dejó

\footnotetext{
20 Donald Coleman menciona que «Las historias individuales de empresas son a la Historia Económica de la empresa lo que las biografias son a la Historia políticam. Ver Donald Coleman (1987), p. 141

${ }^{21}$ Francis E. Hyde (1962), p. 9.

22 Arthur M. Johnson (1962), p. 18.

23 Fritz Redlich (1962), p. 63.
} 
de escribir historia descriptiva de casos especificos y empezó a escribir historia institucional comparada con capacidad para generar conceptos y generalizaciones no históricos» ${ }^{24}$. A pesar de todo, esta transición no fue fácil. La insatisfacción con el sistema de los casos de empresas hizo que los historiadores económicos de la empresa buscasen ayuda en otras ciencias sociales. Según Chandler, la teoría económica en boga en los años cincuenta estaba demasiado orientada hacia el keynesianismo y las técnicas econométricas como para prestar atención a los problemas históricos de la empresa. La conexión entre economistas e historiadores de la empresa había sido más fructifera después de la Primera Guerra Mundial cuando Wesley Mitchell, F. W. Taussig o el mismo John Commons ofrecian al historiador económico de la empresa sus trabajos sobre ferrocarriles, aranceles, sindicatos, bancos o instrumentos financieros ${ }^{25}$. Sin embargo, después de la Segunda Guerra Mundial, la reconexión entre el análisis económico institucional y la Historia Económica de la empresa debía esperar aún una generación más de economistas.

Como todos los historiadores, los «nuevos» historiadores económicos de la empresa estaban interesados en el cambio a lo largo del tiempo. No obstante, su aproximación al estudio del cambio era básicamente distinta al método de los casos. Su objetivo era encontrar no sólo el cuándo y dónde se produjo el cambio, sino también el cómo y el porqué del cambio. Se trataba, en definitiva, de aportar al campo de la Historia Económica de la empresa más síntesis y más análisis del que habia tenido hasta el momento. Esto implicaba, sin embargo, dos cambios estratégicos en el método: uno era la aproximación comparada, otro la aplicación explícita de teoria económica. En cuanto al primero, las obras de Alfred Chandler y Arthur $\mathrm{H}$. Cole sentaron las bases para trabajos posteriores. El primero aportó su conocida triple comparación -entre empresas, por sectores y entre paises - como marco claro para el análisis del desarrollo de la gran empresa multidivisional moderna ${ }^{26}$. El segundo propuso el paradigma del «sistema de negocios» (business system) como red de instituciones mercantiles de una sociedad susceptible de ser analizada en perspectiva comparada para determinar la rapidez y el grado de cambio tecnológico e institucional de unos países con respecto a otros ${ }^{27}$.

El segundo cambio era, no obstante, más difícil. Los usos metodológicos del momento complicaban las cosas. Como señaló Barry Supple, «Clío ha sido siempre una dama mundana tan sensible al dictado de la moda como las otras

\footnotetext{
24 Alfred D. Chandler (1984), p. 3.

25 Ibid., p. 8.

26 Ibid, pp. 11-25.

27 Arthur H. Cole (1962), p. 103.
} 
ciencias sociales» ${ }^{28}$. Además, para llevar a cabo una transformación similar a la que estaba teniendo lugar en la Historia Económica general, la Historia Económica de la empresa necesitaba un armazón teórico que hasta el momento no poseia. De hecho, la evolución de la disciplina en el último cuarto de siglo ha consistido básicamente en añadir nuevas perspectivas teóricas a la experiencia empírica ya existente. Como resultado, este avance teórico ha permitido los mismos logros que en la disciplina-madre, es decir, explicitar algunas teorizaciones implícitas en viejas interpretaciones y derivar otras nuevas a partir de la aplicación simple de teoría económica.

El primer paso en el avance teórico consistió en «recuperar» la teoria económica de la empresa y del empresario. En palabras de Kenneth Boulding, «la economía clásica trataba a la empresa como una entidad nebulosa y al empresario como un personaje aún más indefinido, o al menos oscuro e incluso tenebrosow ${ }^{29}$. La ausencia de un tratamiento detallado del empresario en los economistas clásicos ${ }^{30}$ se convirtió en práctica común en los neoclásicos. En los sistemas de equilibrio general la empresa ejerce el papel implícito de un instrumento input-output sin más función que maximizar la diferencia entre ingresos y costes. Por otra parte, la función empresarial no encuentra un lugar propio y diferenciado, pues el producto total de la economía queda «agotado» al recibir cada factor de producción un pago correspondiente a su aportación marginal. El análisis de Frank Knight «recuperó» al empresario para la tradición neoclásica. En Risk, Uncertainty and Profit (1921) Knight introdujo la diferencia entre riesgo e incertudumbre. El riesgo, según Knight, es simplemente una parte del coste total de la empresa que puede cubrirse a través de un seBuro. La asunción de incertidumbre no asegurable, por el contrario, es la función propia del empresario y la razón de su beneficio al estar vinculada con la habilidad para especular sobre situaciones futuras del mercado. De esta manera, el empresario es el que asume la incertidumbre implicita en cualquier situación futura de equilibrio. El papel *equilibradors del empresario fue reforzado por la obra más reciente de Israel M. Kirzner, Perception, Opportunity and Profit. Estudies in the Theory of Entrepreneurship (1979). Contrariamente a la influencia desequilibradora del empresario schumpeteriano, Kirzner argumenta que:

Dos precios del mismo producto, no explicables por los costes de transacción del mercado, son el resultado de la información imperfecta por parte de los participantes en el mercado. La esencia del proceso empresarial es que una si-

\footnotetext{
28 B. Supple (1958), p. 67.

29. Kenneth E. Boulding (1960), p. 1.

to Véase una discusión al respecto en Mark Blaug (1983), pp. 117.130.
} 
tuación de discrepancia de precios ofrece el incentivo para el arbitraje empresarial y tiende a eliminar la discrepancia ${ }^{31}$.

La recuperación del empresario permitió a los historiadores económicos reintroducir su papel $e$ influencia en los procesos históricos de desarrollo. Refiriéndose a la Revolución Industrial, Barker, Campbell y Mathias afirmaban en 1960 que «estamos educando generaciones de alumnos con una visión tuerta de lo que realmente pasón ${ }^{32}$. La contribución posterior de, entre otros, Sidney Pollard ha tendido a paliar esta situación.

No obstante, los avances teóricos más útiles para el economista histórico de la empresa no se refieren tanto al papel del empresario como a la teoría de la empresa misma. El creciente interés por el crecimiento a largo plazo en el pasado fue un factor esencial. Bien por la propia crisis del keynesianismo o por la creciente crítica a las interpretaciones (muchas veces simplemente implicitas) de los historiadores económicos tradicionales, la atención de los economistas de la historia empezó a centrarse en los mecanismos de oferta como elementos básicos del crecimiento sostenido a largo plazo. La estructura de los mercados y el papel de las empresas en ellos empezó a cobrar protagonismo en un momento de «redescubrimiento" de la teoria de Ronald Coase y de las grandes aportaciones de Edith Penrose, Armen Alchian, Harold Demsetz, John McManus y Oliver Williamson ${ }^{33}$, entre muchisimos otros. La introducción de instrumentos teóricos como la teoria de juegos, el estudio de la actitud frente al riesgo, los costes de transacción, la economía de la información y de la agencia o la economía de los contratos, por ejemplo, ha dado origen a una vasta producción sobre la organización interna de las empresas y sobre su crecimiento, $y$ ha abierto la puerta a toda una serie de investigaciones y reinterpretaciones del papel de la empresa en la historia. Baste șeñalar que el último número (septiembre 1992) del Joumal of Economic Literature menciona al menos treinta articulos recientes cuyo tema central es la empresa y el empresario, y entre los que se cuentan varios de enfoque histórico.

Finalmente, la vieja aspiración de Chandler de reunir el análisis económico institucional con la Historia Económica de la empresa ha sido posible gracias a los avances teóricos de la Nueva Economía Institucional. Los esfuerzos de muchos economistas históricos por incorporar la empresa en las explicaciones de procesos redistributivos han llevado al análisis histórico de monopo-

"Israel M. Kirzner (1979), p. 103.

32 T. C. Barker, R. H. Campbell y P. Mathias (1960), p. 5.

3) Ronald H. Coase (1937), Edith Penrose (1959), Armen A Alchian y Harold Demsetz (1972), John C. McManus (1975), Oliver E. Williamson (1975) y (1988). 
lios, empresas públicas, formación de lobbies empresariales, búsqueda de rentas y regulación estatal. La tradición iniciada por Jonathan Pincus ha sido seguida por Forrest Capie, Bennet D. Baack, Edward John Ray, Stephen B. Webb, Thomas Owen, Frank J. Coppa, entre otros muchos ${ }^{34}$, en un esfuerzo por combinar la Historia Económica con la organización industrial y la economia politica, y pone al alcance de la mano un marco adecuado para explorar el papel de la empresa en la industrialización y el crecimiento. Con esta nueva perspectiva, la Historia Económica de la empresa queda de nuevo vinculada al marco general de la evolución social y política. Así, los estudios históricos de las empresas se ocupan ahora, no sólo de los cambios en la organización interna de la empresa en sí misma, sino también de su impacto sobre aspectos más genetales, tales como la presencia del Estado en la economía o los procesos de industrialización nacional. De esta manera, la nueva Historia Económica de la empresa queda vinculada a la vieja aspiración expresada por Herman Kroos hace tres décadas:

analizar desde una perspectiva histórica el lugar de la empresa en la escena social y económica, y mostrar cómo ha evolucionado en el tiempo el contexto eco. nómico y social que condiciona la organización empresarial ${ }^{35}$.

\section{BIBLIOGRAFLA}

AlChian, Armen A., y Demsetz, Harold (1972): «Production, Information Costs and Economic Organization*, A.E.R., n. 5, diciembre.

BAsck, Bennet D., y RAY, Edward John (1983): «The Political Economy of Tariff Policy: A Case Study of the United Statesw, Explorations in Economic History, XX.

BARKER, T. C.; CAMPBELL, R. H., y MATHIAS, P. (1960): Business History, Londres.

BLAUG, Mark (1983): «Marx, Schumpeter y la teoria del empresariow, Revista de Occidente, V.

Boulding, Kenneth E. (1960): «The Present Position of the Theory of the Firm», en Kenneth E. Boulding y W. Allen Spivey (eds.): Linear Programing and the Theory of the Firm, Nueva York.

CAPIE, Forrest (1983): Depression and Protectionism. Britain Between the Wars, Londres.

CASTEJON, Rafael (1983): aEl empresario schumpeteriano y la historia empresarial», $\mathrm{Pa}$ peles de Economía Española, n. 17.

COASE, Ronald H. (1937): «The Nature of the Firm», Economica, n. 386, noviembre.

COLE, Arthur H. (1962): «What is Business History?», Business History Review, XXXVI, n. 1, primavera.

(1983) Jonathan J. Pincus (1977), Forrest Capie (1983), Bennet D. Baack y Edward John Ray

(1983), Stephen B. Webb (1980), Thomas Owen (1985), Frank J. Coppa (1970).

3s Herman E. Kroos (1962). 
Coleman, D. C., y Mathias, Peter (1984): Enterprise and bistory. Essays in bonour of Charles Wilson, Londres y Nueva York.

Coleman, Donald (1987): «The Uses and Abuses of Business History», Business History, XXIX, n. 2, abril.

CoppA, Frank J. (1970): «The Italian Tariff and the Conflict Between Agriculture and Industry*, The Joumal of Economic History, XXX, diciembre.

ChandleR, Alfred D. (1984): «Comparative business history», en D. C. Coleman y Peter Mathias (eds.) Enterprise and History. Essays in bonor of Charles Wilson, Londres y Nueva York.

Hanna, Leslie (1976): The Rise of the Corporate Economy, Londres.

Harvey, Charles, y Jones, Geoffrey (1990): «Business History in Britain into the 1990 sw, Business History, XXXII, n. 1, enero.

Hidy, Ralph W. (1962): «Henrietta M. Larson: An Appreciation*, Business History Review, XXXVI, n. 1, primavera.

- - (1968), «Business History», International Encyclopedia of the Sacial Sciences, Nueva York y Londres.

HYDE, Francis E. (1962): «Economic Theory and Business History. A Comment on the Theory of Profit Maximization», Business History, V, n. 1, diciembre.

Johnson, Arthur M. (1962): «Where Does Business History Go From Here?, Business History Review, XXXVI, n. 1, primavera.

KIRZneR, Israel M. (1979): Perception, Opportunity and Profit. Estudies in the Theory of Entrepreneurship, Chicago y Londres.

KroOs, Herman E. (1962): «Recruiting Business History Teachers», Business History Review, XXXVI, n. 1, primavera.

LARSON, Henrietta M. (1939): Guide to Business History. Materials for the Study of American Business History and Suggestions for their Use, Cambridge, Massachusetts.

McManus, John C. (1975): «The Costs of Alternative Economic Organizations», Canadian Joumal of Economics, n. 3, agosto.

OWEN, Thomas (1985): «The Russian Industrial Society , The Joumal of Economic History, XLV, septiembre.

PEnRose, Edith (1959): The Theory of the Growth of the Firm, Nueva York.

PINCUS, Jonathan J. (1977): Pressure Groups and Politics in Antebellum Tariffs, Nueva York.

REDLICH, Fritz (1962): «Approaches to Business History», Business History Review, XXXVI, n. 1, primavera.

Supple, B. E. (1958): «American Business History-A Survey», Business History, I, n. 2, junio.

TAWNEY, R. H. (1966): «Introductory Memoir», en R. H. Tawney (ed.), Studies in Economic History: The Collected Papers of George Unwin, (3 ed.), Nueva York.

WaltoN, Clarence C. (1962): «Business History: Some Major Challenges*, Business History Review, XXXVI, n. 1, primavera.

$W_{E B B}$, Stephen B. (1980): *Tariffs, Cartels, Technology, and Growth in the German Steel Industry, 1879-1914*, The Joumal of Economic History, LX, junio.

Williamson, Oliver E. (1975): Markets and Hierarchies: Analysis and Antirrust Implications. $A$ Study in the Economics of Intemal Organization, Nueva York.

- (1988), The Economic Institutions of Capitalism. Fims, Markets, Relational Contracting Nueva York. 